\title{
One-Pot Multicomponent Synthesis of Novel 2-Thioxo- Benzo[6,7]Chromeno[2,3d]Pyrimidin-4-one Derivatives using Cetylpyridinium Chloride
}

Dini Ahanthem, and Warjeet S. Laitonjam*

Department of Chemistry, Manipur University, Canchipur, Imphal -795003, Manipur, India E-mail: Laitonjam Warjeet Singh" warjeet@yahoo.com

\section{ABSTRACT}

The novel 2-thioxo-benzo[5,6]chromeno[2,3- $d$ ]pyrimidin-4-one derivatives were prepared by one pot multicomponent condensation reaction involving thiobarbituric acids, aromatic aldehydes and $\beta$-napthol in acetonitrile-water as solvent using surfactant, cetylpyridinium chloride (CPC) at water bath temperature in good yields. The structures of the compounds were confirmed by elemental analyses and spectral data.

\section{Indexing terms/Keywords}

Multi-component; Chromeno-pyrimidinone; Thiobarbituric acids; CPC

\section{Council for Innovative Research}

Peer Review Research Publishing System

Journal:Journal of Advances in Chemistry

Vol. 7, No. 1

editor@cirworld.com

www.cirworld.com, member.cirworld.com 


\section{INTRODUCTION}

Multi-component, one-pot syntheses have received considerable attention because of their wide range of applications in pharmaceutical chemistry for generation of structural diversity and combinatorial libraries for drug discovery.[1] The use of multicomponent reaction (MCRs) increases due to its flexible, atom economic in nature, operational simplicity and proceed through a sequence of reaction equilibria, yielding the target product.[2] MCRs have also been found to use in synthesizing structurally diverse bioactive heterocyclic compounds.[3] The use of water reduces the harmful organic solvents and is regarded as the greener method in Chemistry.[4] Nitrogen-containing heterocycles are of broad pharmaceutical interest which justifies the continuing efforts in the development of new synthetic strategies.[5]

Chromene derivatives represent an important class of compounds in many natural products [6] and have been reported to possess various pharmacological activities such as antimicrobial,[7] antitumor,[8] antiaggregating,[9] antidepressant[10] and antiproliferative activities.[11] Among the nitrogen containing heterocycles, the chromeno-pyrimidine derivatives are known to possess antiallergic,[12]antimicrobial,[13] antioxidant,[14] anti-inflammatory[15] and anticancer activities.[16] In the field of medicinal chemistry, functionally substituted chromene have been found to play an increasing role in approaching synthetic promising compounds.[17]

As surfactant, at an ambient condition in an aqueous media, aggregates to form micelles with hydrophobic tail and hydrophilic head, micellar surfactants as catalysts are widespread[18] and found to be used in different reactions as a route for synthesis in aqueous solutions.[19] The studies of surfactant-promoted reactions have been increasing, such as, reaction of 1,4-quinone with oxygen nucleophiles in aqeous micelles,[20] surfactant assisted organic reactions in water,[21] Pictet-Spengler reactions.[22]

In view of the above biological activities, various synthetic methods have been reported for the preparation of chromenopyrimidinones derivatives. In continuation of our work on the synthesis of fused heterocyclic compounds derived from thiobarbituric acids,[23]syntheses of 12-aryl-2-thioxo-dihydro-1H-benzo[6,7]chromeno-[3,2-d]pyrimidine-4(12H)-ones in good yields were taken up. Herein, we are reporting the synthesis of the novel chromeno[3,2- $d$ ]pyrimidinones derivatives (2) in one-pot, multicomponent reaction using the surfactant cetylpyridinium chloride (CPC) (Scheme 1). The reactions occur via a three component, one pot reaction between thiobarbituric acid, aldehydes (1) and $\beta$-napthol using CPC in acetonitrile:water (1:1) as solvent, heating at water bath temperature. Addition of the surface active agents in aqueous organic solvent has been known and the use of the surfactant as catalyst has been studied for various organic reactions.

2. General procedure for synthesis of compound (2a). In a typical experiment, $p$-nitrobenzaldehyde $(1.0 \mathrm{mmol}), \beta$-napthol $(1.0 \mathrm{mmol})$, thiobarbituric acid $(1.0 \mathrm{mmol})$ and CPC $(0.015 \mathrm{mmol} \%)$ were taken in a round bottom flask using water and acetonitrile as solvent in 1:1 ratio. The reaction mixture was refluxed for 8 hours in water bath temperature. The reaction was monitored by TLC. After the completion of reaction, the solid was separated and washed with water several times to remove the surfactant and washed again with acetonitrile to remove all the starting substrate to give the pure product, $2 \mathrm{a}(85 \%)$.

5-(4-nitrophenyl)-2-thioxo-2,3-dihydro-1 $\boldsymbol{H - b e n z o [ 6 , 7 ] c h r o m e n o [ 2 , 3 - d ] p y r i m i d i n - 4 ( 5 H ) - o n e ~ ( 2 a ) . ~ Y e l l o w ~ s o l i d ; ~ m . p . ~}$ 207-210 C; IR (KBr): $v_{\max } 3541,1651,1537,1445,1350,1198,1132,1015,849 \mathrm{~cm}^{-1} ;{ }^{1} \mathrm{H}$ NMR (DMSO-d, $\left.300 \mathrm{MHz}\right): \partial$ $5.70(\mathrm{~s}, 1 \mathrm{H}), 6.72(\mathrm{~m}, 1 \mathrm{H}, \mathrm{ArH}), 6.85(\mathrm{~m}, 1 \mathrm{H}, \mathrm{ArH}), 7.01(\mathrm{~d}, 2 \mathrm{H}, \mathrm{J}=7.6 \mathrm{~Hz}, \mathrm{ArH}), 7.24(\mathrm{~m}, 4 \mathrm{H}, \mathrm{ArH}), 7.67(\mathrm{~d}, 2 \mathrm{H}, \mathrm{J}=7.6 \mathrm{~Hz}$, ArH), 10.90 (br s, $1 \mathrm{H}, \mathrm{NH}), 11.20$ (br s, $1 \mathrm{H}, \mathrm{NH}) ;{ }^{13} \mathrm{C}$ NMR (DMSO-d $6.75 \mathrm{MHz}$ ): $\partial$ 173.57, 152.88, 145.70, 128.26, 123.52, 95.62, 31.63; ; HRMS (El) cald for $\mathrm{C}_{25} \mathrm{H}_{23} \mathrm{~N}_{3} \mathrm{O}_{4} \mathrm{~S}$ : 461.53; found 461.104.

5-(4-chlorophenyl)-2-thioxo-2,3-dihydro-1 $\boldsymbol{H}$-benzo[6,7]chromeno[2,3- $d$ ]pyrimidin-4(5H)-one(2b): White solid; m.p: $198-203^{0} \mathrm{C}$; IR (KBr): $v_{\max } 3524,1660,1537,1443,1359,1201,1134,1013,866 \mathrm{~cm}^{-1} ;{ }^{1} \mathrm{H}$ NMR (DMSO-d, $\left.300 \mathrm{MHz}\right): \partial$ $5.80(\mathrm{~s}, 1 \mathrm{H}), 6.97-7.22(\mathrm{~m}, 10 \mathrm{H}, \mathrm{ArH}), 11.32(\mathrm{br} \mathrm{s}, 1 \mathrm{H}, \mathrm{NH}), 11.69$ (br s, $1 \mathrm{H}, \mathrm{NH}) ;{ }^{13} \mathrm{C}$ NMR (DMSO-d, $\left.75 \mathrm{MHz}\right): 2173.37$, 164.01, 163.03, 142.73, 129.78, 128.93, 128.01, 95.98, 30.60; HRMS (El) cald for $\mathrm{C}_{21} \mathrm{H}_{13} \mathrm{CIN}_{2} \mathrm{O}_{2} \mathrm{~S}: 393.0464$; found 393.0478.

5-(1H-indol-3-yl)-2-thioxo-2,3-dihydro-1 $\boldsymbol{H}$-benzo[6,7]chromeno[2,3- $d]$ pyrimidin-4(5H)-one(2c): Orange solid; m.p: 188-192 ${ }^{\circ} \mathrm{C}$; IR (KBr): $v_{\max } 3538,1655,1528,1377,1277,1213,1157 \mathrm{~cm}^{-1} ;{ }^{1} \mathrm{H}$ NMR (DMSO-d, $\left.300 \mathrm{MHz}\right): \partial 5.80(\mathrm{~s}, 1 \mathrm{H})$, 6.97-7.22 (m, 10H, ArH), 12.28 (br s, $1 \mathrm{H}, \mathrm{NH}), 12.32$ (br s, $1 \mathrm{H}, \mathrm{NH}), 13.01$ (br s, $1 \mathrm{H}, \mathrm{NH}) ;{ }^{13} \mathrm{C}$ NMR (DMSO-d, $\left.75 \mathrm{MHz}\right): \partial$ $178.19,163.26,161.46,145.05,141.54,137.11129 .54,124.53,123.59,118.35,113.86,112.88,109.23,29.50 ;$ HRMS (El) cald for $\mathrm{C}_{21} \mathrm{H}_{13} \mathrm{ClN}_{2} \mathrm{O}_{2} \mathrm{~S}: 304.3127$; found 304.3238 . 
5-(3-hydroxynaphthalen-1-yl)-2-thioxo-2,3,4a,5-tetrahydro-1 $H$-benzo[6,7]chromeno[2,3-d]pyrimidin-4(12a $H$ one(2d): Reddish Orange solid; m.p: $195-198^{0} \mathrm{C}$; IR (KBr): $v_{\max } 3452,3032,1678,1564,1458,1211,1138,814 \mathrm{~cm}^{-1} ;{ }^{1} \mathrm{H}$ NMR (DMSO-d, $300 \mathrm{MHz})$ : $25.56(\mathrm{~s}, 1 \mathrm{H}), 7.15(\mathrm{~m}, 2 \mathrm{H}, \mathrm{ArH}), 7.22(\mathrm{~m}, 2 \mathrm{H}, \mathrm{ArH}), 7.55(\mathrm{~m}, 2 \mathrm{H}, \mathrm{ArH}), 7.60(\mathrm{~m}, 2 \mathrm{H}, \mathrm{ArH})$, $7.88(\mathrm{~m}, 2 \mathrm{H}, \mathrm{ArH}), 8.22(\mathrm{~m}, 2 \mathrm{H}, \mathrm{ArH}), 11.55$ (br s, $1 \mathrm{H}, \mathrm{NH}), 12.20$ (br s, $1 \mathrm{H}, \mathrm{NH}) ;{ }^{13} \mathrm{C}$ NMR (DMSO-d, $\left.75 \mathrm{MHz}\right): \partial 174.88$, 173.46, 160.25, 156.78, 148.25, 131.42, 129.80, 128.04, 126.61, 126.49, 123.14, 119.11, 109.14, 43.16, 29.56; HRMS (El) cald for $\mathrm{C}_{25} \mathrm{H}_{18} \mathrm{~N}_{2} \mathrm{O}_{3} \mathrm{~S}: 426.1038$; found 426.4870 .

5-(4-dimethylaminophenyl)-2-thioxo-2,3-dihydro-1 H-benzo[6,7]chromeno[2,3-d]pyrimidin-4(5H)-one(2e): Redish orange solid; m.p: 205-208 ${ }^{0} \mathrm{C}$; IR (KBr): $\mathrm{v}_{\max } 3457,3119,1634,1537,1495,1373,1194,1142,1011 \mathrm{~cm}^{-1} ;{ }^{1} \mathrm{H} \mathrm{NMR}$ (DMSO-d $\left.\mathrm{d}_{6}, 300 \mathrm{MHz}\right): \partial 3.00\left(\mathrm{~s}, 3 \mathrm{H}, \mathrm{NCH}_{3}\right), 3.02\left(\mathrm{~s}, 3 \mathrm{H}, \mathrm{NCH}_{3}\right), 5.90(\mathrm{~s}, 1 \mathrm{H}), 6.75(\mathrm{~m}, 2 \mathrm{H}, \mathrm{ArH}), 7.22(\mathrm{~m}, 2 \mathrm{H}, \mathrm{ArH})$, $7.40(\mathrm{~m}, 2 \mathrm{H}, \mathrm{ArH}), 8.22(\mathrm{~m}, 2 \mathrm{H}, \mathrm{ArH}), 8.48(\mathrm{~m}, 2 \mathrm{H}, \mathrm{ArH}), 11.98(\mathrm{br} \mathrm{s}, 1 \mathrm{H}, \mathrm{NH}), 12.05$ (br s, $1 \mathrm{H}, \mathrm{NH}) ;{ }^{13} \mathrm{C} \mathrm{NMR}\left(\mathrm{DMSO}-\mathrm{d}_{6}\right.$, $75 \mathrm{MHz}$ ): $\partial$ 178.02, 173.46, 163.54, 160.98, 156.66, 155.26, 140.25, 128.62, 120.92, 112.01, 109.83, 95.92, 46.09, 30.86; HRMS (El) cald for $\mathrm{C}_{23} \mathrm{H}_{19} \mathrm{~N}_{3} \mathrm{O}_{2} \mathrm{~S}: 401.1198$; found 401.4809 .

5-(4-carbaldehydophenyl)-2-thioxo-2,3-dihydro-1 $\boldsymbol{H}$-benzo[6,7]chromeno[2,3- $d]$ pyrimidin-4(5H)-one(2f): Orange solid; m.p: $201-203^{0} \mathrm{C}$; IR (KBr): $v_{\max } 3522,3148,1670,1574,1518,1433,1298,1207,1148 \mathrm{~cm}^{-1}$; ${ }^{1} \mathrm{H}$ NMR (DMSO-d 6 , $300 \mathrm{MHz}$ ): $\partial 4.50$ (s, 1H), 5.90 (s, 1H), 7.12-8.25 (m, 10H, ArH), 11.62 (br s, 1H, CHO), 12.25 (br s, $1 \mathrm{H}, \mathrm{NH}), 12.42$ (br s, $1 \mathrm{H}, \mathrm{NH}) ;{ }^{13} \mathrm{C}$ NMR (DMSO-d 6 , $75 \mathrm{MHz}$ ): $\partial$ 178.42, 173.50, 162.53, 145.23, 132.70, 128.60, 61.30, 29.56; HRMS (El) cald for $\mathrm{C}_{22} \mathrm{H}_{14} \mathrm{~N}_{2} \mathrm{O}_{3} \mathrm{~S}: 386.4232$; found 386.0725.

5-Propyl-2-thioxo-2,3-dihydro-1 H-benzo[6,7]chromeno[2,3-d]pyrimidin-4(5H)-one(2g): Blacksolid; m.p: $188-192^{0} \mathrm{C}$; IR $(\mathrm{KBr}): \mathrm{v}_{\max } 3178,1686,1582,1522,1316,1165 \mathrm{~cm}^{-1} ;{ }^{1} \mathrm{H}$ NMR (DMSO-d, $\left.300 \mathrm{MHz}\right): \partial 0.82\left(\mathrm{~m}, 3 \mathrm{H}, \mathrm{CH}_{3}\right), 1.18(\mathrm{~m}, 2 \mathrm{H}$, $\left.\mathrm{CH}_{2}\right), 2.28\left(\mathrm{~m}, 2 \mathrm{H}, \mathrm{CH}_{2}\right), 5.78(\mathrm{~m}, 1 \mathrm{H}), 7.12(\mathrm{~m}, 2 \mathrm{H}, \mathrm{ArH}), 7.32-7.45(\mathrm{~m}, 2 \mathrm{H}, \mathrm{ArH}), 7.65-7.72(\mathrm{~m}, 2 \mathrm{H}, \mathrm{ArH}), 11.98(\mathrm{br} \mathrm{s}, 1 \mathrm{H}$, $\mathrm{NH}$ ), 12.05 (br s, $1 \mathrm{H}, \mathrm{NH}) ;{ }^{13} \mathrm{C}$ NMR (DMSO-d, $75 \mathrm{MHz}$ ): $\partial$ 178.22, 157.26, 145.48, 135.25129.79, 128.60, 128.04, 126.60, 126.48, 123.13, 119.10, 109.13, 31.81, 29.56, 22.43, 14.48; HRMS (EI) cald for $\mathrm{C}_{18} \mathrm{H}_{16} \mathrm{~N}_{3} \mathrm{O}_{2} \mathrm{~S}: 324.0932$; found 324.3968 .

5-(2,4-dimethoxyphenyl)-2-thioxo-2,3,4a,5-tetrahydro-1 H-benzo[6,7]chromeno[2,3-d]pyrimidin-4(12a H)-one(2h): White solid; m.p: $185-187^{0} \mathrm{C}$; IR (KBr): $\mathrm{v}_{\max } 3181,1688,1576,1543,1495,1379,1260,1175,1034,949 \mathrm{~cm}^{-1} ;{ }^{1} \mathrm{H} \mathrm{NMR}$ (DMSO-d, $300 \mathrm{MHz}): \partial 3.73\left(\mathrm{~s}, 3 \mathrm{H}, \mathrm{OCH}_{3}\right), 3.85\left(\mathrm{~s}, 3 \mathrm{H}, \mathrm{OCH}_{3}\right), 4.56(\mathrm{~s}, 1 \mathrm{H}), 7.08-7.23(\mathrm{~m}, 5 \mathrm{H}, \mathrm{ArH}), 7.40-8.13(\mathrm{~m}, 4 \mathrm{H}$, ArH), 12.34 (br s, $1 \mathrm{H}, \mathrm{NH}), 12.48$ (br s, $1 \mathrm{H}, \mathrm{NH}) ;^{13} \mathrm{C}$ NMR (DMSO-d, $\left.75 \mathrm{MHz}\right): \partial$ 178.49, 161.73, 159.44, 155.17, 154.09, $150.11,145.35,129.24,127.99,126.06,125.90,122.59,121.59,121.17,118.39,116.98,56.25,55.46,28.84$; HRMS (EI) cald for $\mathrm{C}_{23} \mathrm{H}_{20} \mathrm{~N}_{2} \mathrm{O}_{4} \mathrm{~S}: 420.4809$; found 420.1144 .

\section{Results and discussion}

For optimization of reaction condition, various surfactants such as sodium dodecylsulphate (SDS), cetyltrimethylammonium bromide (CTAB), cetylpyridinium chloride (CPC), tetradecyltrimethyl ammonium bromide (TTAB), TEAB were employed as catalyst in water alone and in water:acetonitrile (1:1) as solvent for the reaction of thiobarbituric acid, $p$-nitrobenzaldehyde and $\beta$-napthol (table 1). No expected product was observed when SDS (1 mmol) was used in water (entry 1). When SDS (1 mmol) was employed as catalyst in water:acetonitrile (1:1) solvent, the product $2 a$ was afforded in $42 \%$ yield on refluxing at water bath temperature (entry 4 ); however, expected product was not obtained on stirring at room temperature (entry 2) and at higher temperatures (entry 3 ). While TTAB, TEAB and CTAB afforded around $60-65 \%$ overall yield (entries 5, 6 \& 8); CPC (1 mmol) gave $85 \%$ yield (entry 7$)$. On taking CPC (1.5 mmol) as catalyst in water:acetonitrile (1:1) ass solvent, 2 a was obtained as $82 \%$ yield (entry 9 ), and reaction in water afforded $53 \%$ yield using CPC $(1.0 \mathrm{mmol})$ as catalyst by refluxing for $24 \mathrm{hr}$ (entry 10). The influence of the solvent system was further evaluated and the usage of a medium-polarity solvent acetonitrile which is miscible with water is used in various ratios with water as solvent. It can be seen that the use of the mixture of acetonitrile in water can enhance the solubility of the substrate and increases the yield of the product $2 a^{16}$ Thus, from the above observation, it was found that treatment of thiobarbituric acid, $p$-nitrobenzaldehyde and $\beta$-napthol using CPC as catalyst in water:acetonitrile (1:1) on refluxing at water bath temperature afforded the desired product, 2a significantly (85\%).

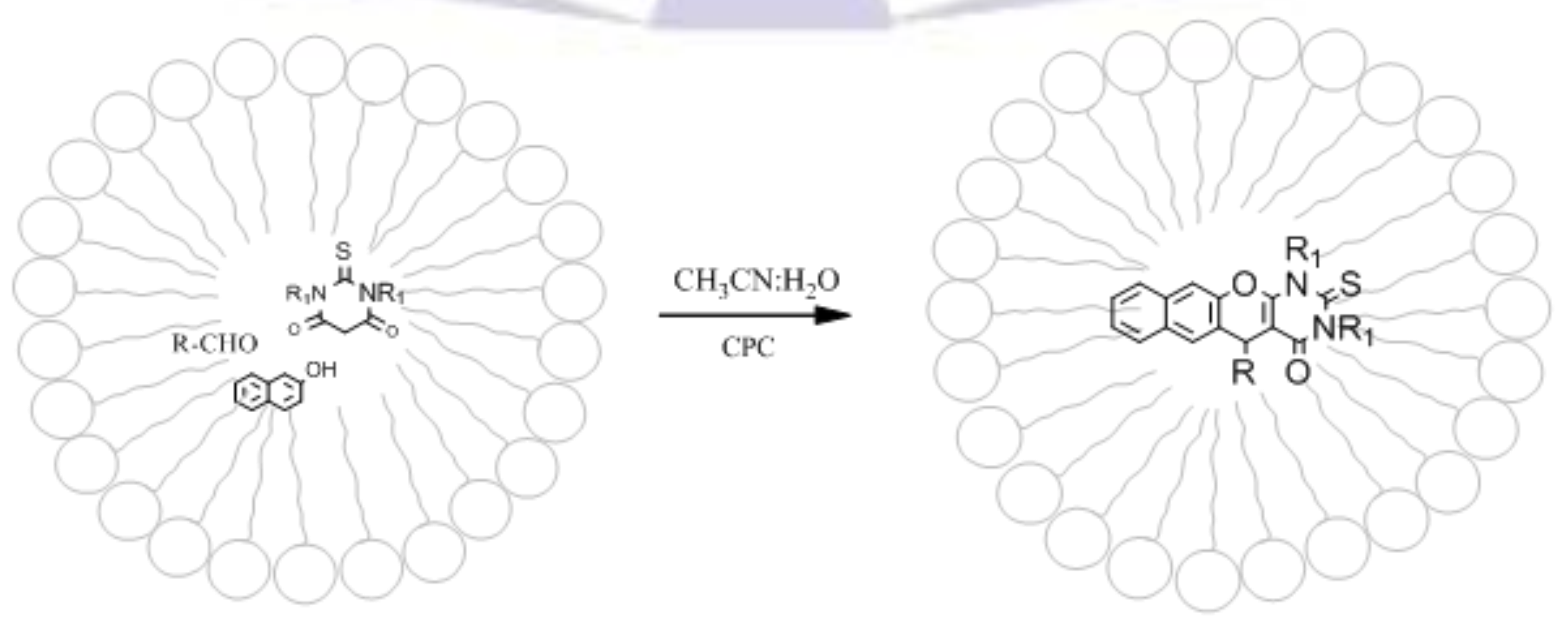

Fig.1. Schematic diagram illustrating the role of CPC 


\section{Table 1: Optimization of reaction condition}

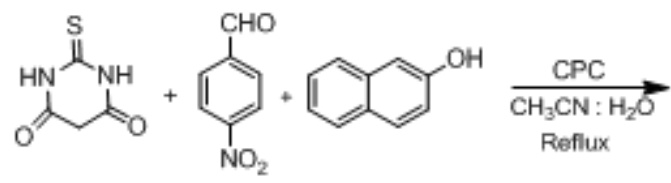<smiles>O=c1[nH]c(=S)[nH]c2c1C(c1ccc([N+](=O)[O-])cc1)c1cc3ccccc3cc1O2</smiles>

$2 \mathrm{a}$

\begin{tabular}{llllll}
\hline Entry & Surfactant & Solvent & Temperature & Time (hr) & 2a (\%) \\
\hline 1 & SDS $(1 \mathrm{mmol})$ & $\mathrm{H}_{2} \mathrm{O}$ & R.T & - & - \\
2 & $\mathrm{SDS}(1 \mathrm{mmol})$ & $\mathrm{CH}_{3} \mathrm{CN}: \mathrm{H}_{2} \mathrm{O}$ & R.T & - & - \\
3 & $\mathrm{SDS}(1 \mathrm{mmol})$ & $\mathrm{CH}_{3} \mathrm{CN}: \mathrm{H}_{2} \mathrm{O}$ & $80^{\circ} \mathrm{C}$ & - & - \\
4 & $\mathrm{SDS}(1 \mathrm{mmol})$ & $\mathrm{CH}_{3} \mathrm{CN}: \mathrm{H}_{2} \mathrm{O}$ & Reflux & 24 & 42 \\
5 & $\mathrm{TTAB}(1 \mathrm{mmol})$ & $\mathrm{CH}_{3} \mathrm{CN}: \mathrm{H}_{2} \mathrm{O}$ & Reflux & 18 & 60 \\
6 & $\mathrm{CTAB}(1 \mathrm{mmol})$ & $\mathrm{CH}_{3} \mathrm{CN}: \mathrm{H}_{2} \mathrm{O}$ & Reflux & 20 & 65 \\
7 & $\mathrm{CPC}(1 \mathrm{mmol})$ & $\mathrm{CH}_{3} \mathrm{CN}: \mathrm{H}_{2} \mathrm{O}$ & Reflux & 12 & 85 \\
8 & $\mathrm{TEAB}(1 \mathrm{mmol})$ & $\mathrm{CH}_{3} \mathrm{CN}: \mathrm{H}_{2} \mathrm{O}$ & Reflux & 14 & 65 \\
9 & $\mathrm{CPC}(1.5 \mathrm{mmol})$ & $\mathrm{CH}_{3} \mathrm{CN}: \mathrm{H}_{2} \mathrm{O}$ & Reflux & 12 & 82 \\
10 & $\mathrm{CPC}(1 \mathrm{mmol})$ & $\mathrm{H}_{2} \mathrm{O}$ & Reflux & 24 & 53 \\
\hline
\end{tabular}

From the observation of the optimization studies, the multi-component reaction (MCR) of this greener system was further studied to a variety of thiobarbituric acid and aldehydes (1) with $\beta$-napthol using CPC as catalyst in water:acetonitrile (1:1) on refluxing at water bath temperature to yield 2 (table 2). The formation of $\mathbf{2}$ is assumed to proceed through the in situ intramolecular cyclization of the intermediate $[\mathrm{A}]$ which is formed by nucleophilic addition of $\beta$-napthol to the condensation product of thiobarbituric acid and aldehyde (scheme 2). We have observed that the reaction was more favourable with the electron withdrawing group and less facile with the electron releasing group.

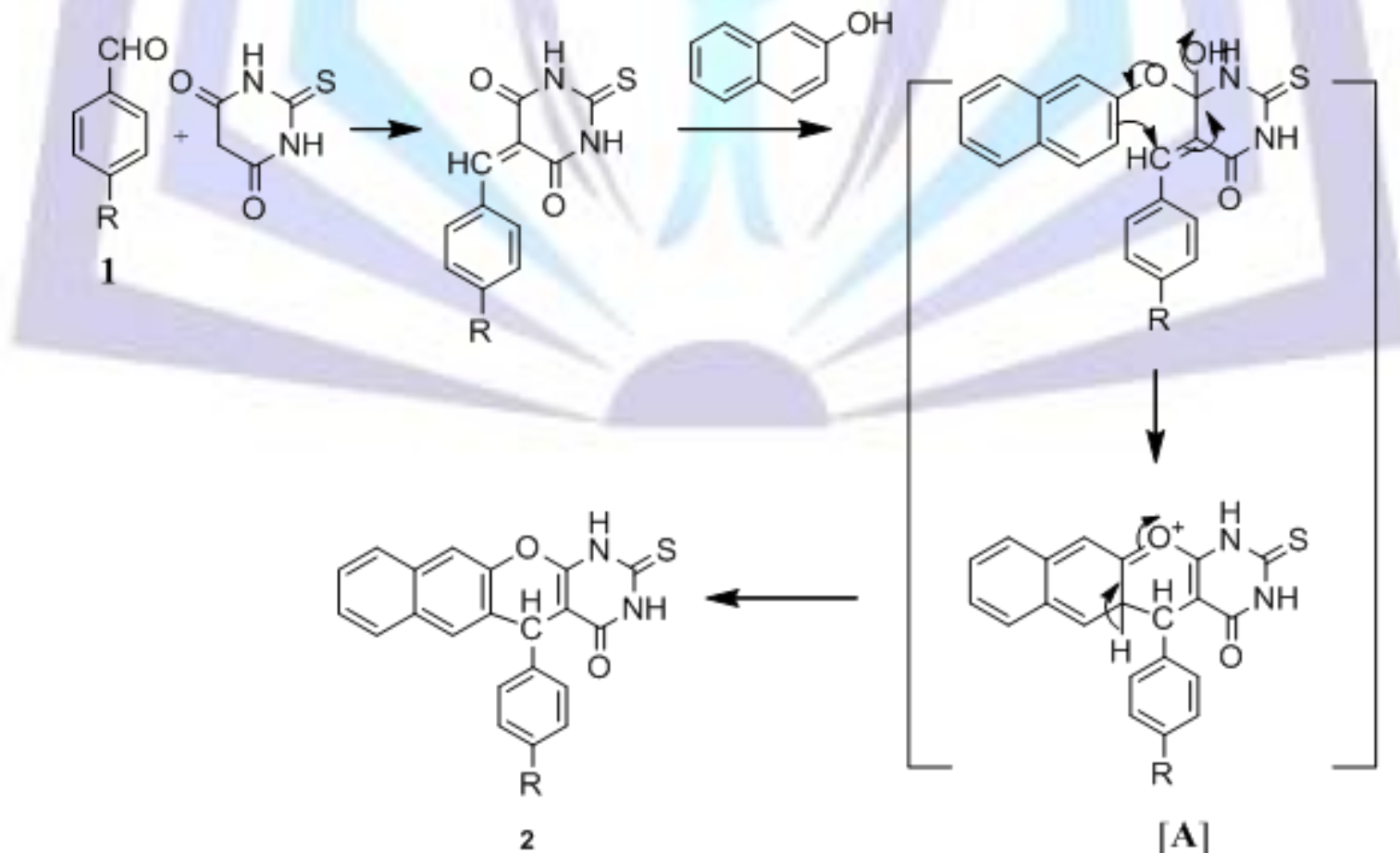

Scheme 2 
Table 2: Reaction of thiobarbituric acid with aldehydes (1) to give the products, 2.<smiles>O=C1CC(=O)NC(=S)N1</smiles><smiles>[R]C=[O+]c1ccc2ccc(O)cc2c1</smiles>

1

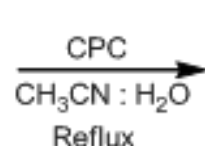
Reflux<smiles>[R]C1c2cc3ccccc3cc2Oc2[nH]c(=S)[nH]c(=O)c21</smiles>

2

\section{Entry}

Aldehyde (1)

Product (2)<smiles>O=c1[nH]c2c(c(=O)[nH]1)C(c1ccc([N+](=O)[O-])cc1)c1cc3ccccc3cc1O2</smiles>

1<smiles>O=Cc1ccc([N+](=O)[O-])cc1</smiles>

2

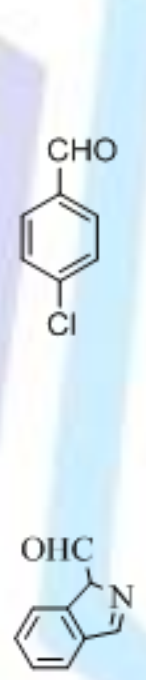

3

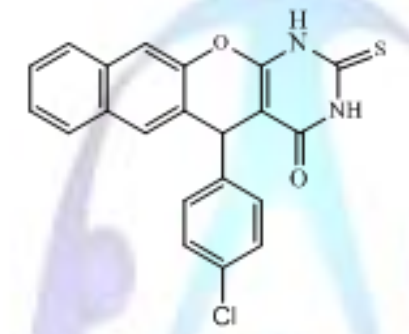

10<smiles>O=c1[nH]c(=S)[nH]c2c1C(C1N=Cc3ccccc31)c1cc3ccccc3cc1O2</smiles>

$85 \quad 207-210$

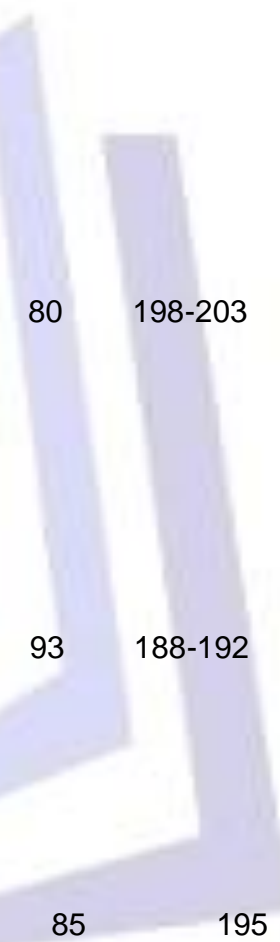

4

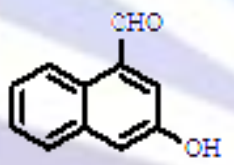<smiles>O=C1NC(=S)NC2Oc3cc4ccccc4cc3C(c3cc(O)cc4ccccc34)C12</smiles>

4

3

195

5<smiles></smiles><smiles>CNc1ccc(C2c3cc4ccccc4cc3Oc3[nH]c(=S)[nH]c(=O)c32)cc1</smiles>

4

85

205-208

6<smiles>O=Cc1ccc(C=O)cc1</smiles><smiles>O=Cc1ccc(C2c3cc4ccccc4cc3Oc3[nH]c(=S)[nH]c(=O)c32)cc1</smiles>

4

$92 \quad 201-203$ 
7

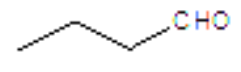

8<smiles>CCCC1c2cc3ccccc3cc2Oc2[nH]c(=S)[nH]c(=O)c21</smiles>

5

75

188-192

\section{Conclusion}

In conclusion, an efficient way of synthesising substituited chromeno pyrimidine derivatives in good yields was developed. A series of chromeno pyrimidine derivatives which were able to synthesise from this greener one pot multi component reaction of thiobarbituric acid, aromatic aldehydes, $\beta$-napthol using surfactant and acetonitrile:water as solvent has been described. The reaction products were characterised by IR, ${ }^{1} \mathrm{H}$ NMR, ${ }^{13} \mathrm{C}$ NMR \& Mass spectral data.

\section{REFERENCES}

[1] Zhu, J.; Bienayme, H. Multi-Component Reactions; Wiley: Weinheim, 2005.

[2] (a) Lu, P.; Wang, Y. G. Synlett 2010, 165-173; (b) Ganem, B. Acc. Chem. Res. 2009, 42, 463-472; (c) Dömling, A. Chem. Rev. 2006, 106, 17-89; (d) Ramo' n, D. J.; Yus, M. Angew. Chem., Int. Ed. 2005, 44, 1602-1634; (e) Orru, R. V. A.; de Greef, M. Synthesis 2003, 1471-1499; (f) Ugi, I.; Heck, S. Comb. Chem. High Throughput Screening 2001, 4, 1-34; (g) Weber, L.; Illgen, K.; Almstetter, M. Synlett 1999, 366-374; (g) Wender, P.A.; Handy, S.T.; Wright, D.L. Chem. Ind. 1997,765; (h) Trost, B.M. Angew. Chem. Int.Ed. Engl. 1995, 34, 259.

[3] (a) Domling, A. Chem. Rev. 2006,106,17-89; (b) Multicomponent Reactions; Zhu, J., Bienayme, H., Eds. Wiley,VCH, Wienheim, 2005.; (b) Ramon,D.J.; Yus, M. Angew. Chem., Int. Ed. 2005, 44, 1602-1634

[4] Tundo, P.; Black, D. S. C.; Breen, J.; Collins T.; Memoli, S.; Miyamato, J.; Polyakoff, M; Tumas, W. Pure Appl. Chem. 2000, 72, 1207.

[5] Abass, M.; Ismail, M. M.; Abdel-Monem, W. R.; Mayas, A. S. J. Serb. Chem. Soc. 2010, 75(1), 11.

[6] (a) J. Kuthan, Adv. Heterocycl. Chem. 1983, 34, 145; (b) S. Hatakeyama, N. Ochi, H. Numata, et al. Chem. Commun. 1988, 17, 1202.

[7] M.P. Georgiadis, E.A. Cauladouros, A.K. Delitheos, J. Pharm. Sci. 81 (1992) 1126.

[8] S.J. Mohr, M.A. Chirigos, F.S. Fuhrman. Cancer Res. 35 (1975) 3750.

[9] A. Bargagna, M. Longobardi, E. Mariani. Farmaco 46 (1991) 461.

[10] A. Ermili, G. Roma, M. Buonamici. Farmaco Ed. Sci. 34 (1979) 535.

[11] M. Brunavs, C.P. Dell, P.T. Gallagher. Chem. Abstr. 120 (1994) $106768 t$.

[12] M. Ban, H. Taquchi, T. Katsushimal. Bioorg. Med. Chem. 6 (1998) 1057.

[13] (a) B.K. Pankaj, H.C. Kishor, K.S. Nisha, et al. Arkivoc xi (2009) 326;(b) L. Jian-Chao, H. Hong-Wu, C. He-Lian, Chin. J. Org. Chem. 8 (2011) 1208.

[14] C. Milan, M. Maja, S. Bojan,. Molecules 15 (2010) 6795.

[15] Y. Bansal, S. Ratra, G. Bansal. J. Iran. Chem. Soc. 6 (2009) 504.

[16] S. Kamaljit, S. Kawaljit, W. Baojie, Eur. J. Med. Chem. 46 (2011) 2290.

[17] (a) Stachulski,A.V.;Berry, N.G.; Low,A.C.L.;Moores, S.L.;Row,E;Warhurst, D.C.;Adagu, I.S.; Rossignol, J-F. J.Med. $\quad$ Chem.Lett.2006,49,1450. $\quad$ (b) Gesson,J.P.;Fonteneau, N.;Mondon,M.;Charbit,S.;Ficheux,H.;Schutze,F.US patent 6,965,039 B2,2005. (c) Sun,W.;Cama, L.J.; Birzin, E.T.;Warrier, S.;Locco, L; Mosley, R.;Hammond,M.L.;Rohrer, S.P Bioorg. Med.Chem.2006,16,1468.

[18] (a) Kobayashi, S. Chem. Lett. 1991, 2187-2190; (b) Kobayashi, S.; Hachiya, I. J. Org. Chem. 1994, 3950-3596; (c) Larpent, C.; Bernard, E.; Menn, F.B.; Patin, H. J. Mol. Catal. A: Chem. 1997, 116, 227-228; (d) Firouzabadi, H.; Iranpoor, N.; Garzan, A. Adv. Synth. Catal. 2005, 347, 1925-1928.

[19] Van den Broeke, L. J. P.; de Bruijin, V.G.; Heijnen, J. H. M.; Keurentjes, J. T. F. Ind. Eng. Chem. Res. 2001, 40, 5240-5245. 
[20] Tandon.V.K.; Maurya H.K. Tetrahedron Letters 2010, 51, 3843-3847.

[21] Vanden Eynde J.J.; Mutonkole K.; Haverbeke,Y.V. Ultasonics Sonochemistry 2001, 8, 35-39.

[22] Saito. A.; Takayama, M.; Yamazaki, A.; Numaguchi , J.; Hanzawa, Y.; Tetrahedron 2007, 63, 4039-4047.

[23] (a) Nongmeikapam, A. D.; Laitonjam, W.S. Ind. J. Chem. 1994, 33B, 1091-1092; (b) Nongmeikapam, A. D.; Laitonjam, W.S. Ind. J. Chem. 1996, 35B, 478-479; (c) Thokchom. H.S.; Nongmeikapam, A. D.; Laitonjam, W.S. Can. J. Chem. 2005, 83, 1056-1062; (d) Moirangthem, N.D.; Laitonjam, W.S. Beil. J. Org. Chem. 2010, 6, 10561060. 